\title{
Dose distributions verification for High dose rate brachytherapy plans by using ionization chambers 2D array
}

\author{
${ }^{1}$ R. Moustafa Abdelfattah, ${ }^{2} \mathrm{~N}$. Ahmed Deiab , ${ }^{3} \mathrm{M}$. Hassan Elnaggar, ${ }^{4} \mathrm{M}$. Hany Khedr, ${ }^{5} \mathrm{R}$. \\ Abdelmoneim Rizk \\ ${ }^{1}$ Medical Physicist , Radiotherapy and Nuclear Medicine Department, National Cancer Institute of Cairo \\ University \\ rasha_Amylee@hotmail.com \\ ${ }^{2}$ Assistant professror of medical physics Physicist, Radiotherapy and Nuclear Medicine Department, National \\ Cancer Institute of Cairo University \\ nashaatad@hotmail.com \\ ${ }^{3}$ Professor of Radiation Oncology, Radiation Oncology department, National cancer institute of Cairo University. \\ mervatelnaggar@hotmail.com \\ ${ }^{4}$ Lecturer of Biomedical Physics, Physics Department,Faculty of Science of Helwan University \\ mhkdahawy@yahoo.com \\ ${ }^{5}$ Professor of Radiation physics, Physics Department,Faculty of Science of Helwan University
}

\section{ABSTRACT} rizk1953@live.com

\begin{abstract}
The purpose of this paper is to perform dosimetric verification (in-phantom) of dose distributions calculated with treatment planning system (TPS) by using 2D chamber array device in HDR brachytherapy. HDR brachytherapy treatment plans'dose distribution verification is performed using the two-dimensional (2D) ionization chamber array MatriXX Evolution developed by IBA Dosimetry (IBA Dosimetry, Germany) whose detector area is covered with the Nucletron Freiburg Flap Applicator Set (Nucletron BV,Veenendaal, the Netherlands) with catheters such that the detector plane was set to $0.86 \mathrm{~cm}$ from the catheter plane. Fixed slabs of RW3 (Perspex) were added below the 2D-ARRAY to provide full scattering conditions. The phantom was scanned on computed tomography (CT) for treatment planning with $2.5-\mathrm{mm}$ slice thickness. Based on the CT data of the phantom, three different plans were calculated by the planning system (Oncentrabrachy version 4.3, Nucletron BV) and then are exported to the VERISOFT software for comparison with measured data. For comparison of dose distributions, both dose planes - measured \& calculated - were normalized to the global maximum dose of the reference matrix (measured data set) and compared using the Gamma index method. Gamma indexes were evaluated using a dose-difference criterion of $3 \%$ and a distance criterion of $3 \mathrm{~mm}(\gamma \leq 1)$. The total number of evaluated dose points for the vault test case is $9755,98.6 \%$ of them (9623 point) passed the criteria of acceptability ( $3 \%$ delta dose and $3-\mathrm{mm}$ distance criteria) and $1.4 \%$ of them (132 point) failed it. The total number of evaluated dose points for the full test case is $19964,93.6 \%$ of them (18683 point) passed the criteria of acceptability and $6.4 \%$ of them (1281 point) failed it. And the total number of evaluated dose points for the cylinder test case is $19871,96.9$ $\%$ of them (19258 point) passed the criteria of acceptability and 3.1\% of them (613 point) failed it. By thisThe use of the two-dimensional (2D) ionization chamber array MatriXX Evolution for brachytherapy applications has been successfully demonstrated. The array measurements in these planes have shown acceptable agreement with the TPS, generally within $3 \%$ delta dose and 3-mm distance agreement criteria within each plane. The comparisons made led to make a relation between the passing percentage values to the number of the evaluated dose points for each test case and it was found that as the number of these pixels increases the possibility of having more failing points increases.
\end{abstract}

\section{Council for Innovative Research}

Peer Review Research Publishing System

\section{Journal: INTERNATIONAL JOURNAL OF COMPUTERS \& TECHNOLOGY}

Vol. 15, No. 3

www.ijctonline.com, editorijctonline@gmail.com 


\section{Indexing terms/Keywords}

HDR Brachytherapy; dose distribution; verification; dosimetry; treatment planning

\section{Academic Discipline And Sub-Disciplines}

Computer Science, Computer Engineering, Information Technology, Information Systems

\section{SUBJECT CLASSIFICATION}

Pattern Recognition, Artificial Intelligence, Machine Learning

\section{TYPE (METHOD/APPROACH)}

Experimentation, Empirical studies, Computer Simulation

\section{INTRODUCTION}

The efficacy of radiation therapy relies on the accuracy of dose delivery to patients. Proper implementation of a treatment planning system for accurate treatment dose calculations and quality assurance procedures to detect dosimetric errors are of critical importance. Since the Brachytherapy treatment planning systems use simplified algorithmsin their dose calculations, verification of the dose distributions presented by the systems may be of clinical importance. Evaluation of the treatment planning systems in terms of their presented dose distribution can be a part of quality assurance in the clinical practice of brachytherapy. The dose distribution generated by the TPS using the AAPM TG-43 dose formalism [1] is usually compared with the calculations using the Sievert summation [2], Monte Carlo simulation [3], or dose distributions measured with Gafchromic film [4]. Gafchromic film is an excellent tool for dosimetry but involves a consumable cost, requires characterization of both the filmand the scanner, and presents several technical challenges in yielding accurate dosimetric results [5]. Recently, 2D ionization chamber arrays have become increasingly popular for intensity-modulated radiation therapy (IMRT) and volumetric-modulated arc therapy (VMAT) plan verification. The detector was found to be linear with dose, independent of dose rate,and a suitable device for quality assurance and $2 \mathrm{D}$ dose verifications of megavoltage beams.

\section{MATERIALS AND METHOD}

In this study, Nucletron (Nucletron, Veenendaal, The Netherlands) MicroSelectron remote-afterloading HDR brachytherapy 30 channels unit (for treatment applicator connection) fed with Nucletron Ir-192 HDR-v2r (new design) radiation source is used to deliver the prescribed radiation dose planned by Oncentra-Master-Plan-Brachy treatment planning system whose calculation algorithm is based on AAPM TG-43 dose formalism. The dosimetric verification is performed using the two-dimensional (2D) ionization chamber array MatriXX Evolution developed by IBA Dosimetry (IBA Dosimetry, Germany). The MatriXX Evolution consists of 1020 vented, plane-parallel cylindrical ionization chambers arranged in a $32 \times 32$ matrix with a maximum field-of-view of $24.4 \times 24.4 \mathrm{~cm}^{2}$. The chamber size is $4.5-\mathrm{mm}$ diameter and 5 - $\mathrm{mm}$ height, center-to-centerdistance is $0.76 \mathrm{~cm}$, active volume is $0.08 \mathrm{~cm}^{3}[6,7]$. The detector area is covered with the Nucletron Freiburg Flap Applicator Set (Nucletron BV,Veenendaal, the Netherlands)with catheters such that the detector plane was set to $0.86 \mathrm{~cm}$ from the catheter plane [8]. The Freiburg Flap Applicator Set shown in figurer 1 is a flexible mesh style surface mold made of silicone rubber with 36 channels for implant tubeswith a separation of $10 \mathrm{~mm}$ and a channel length of $24 \mathrm{~cm}$.

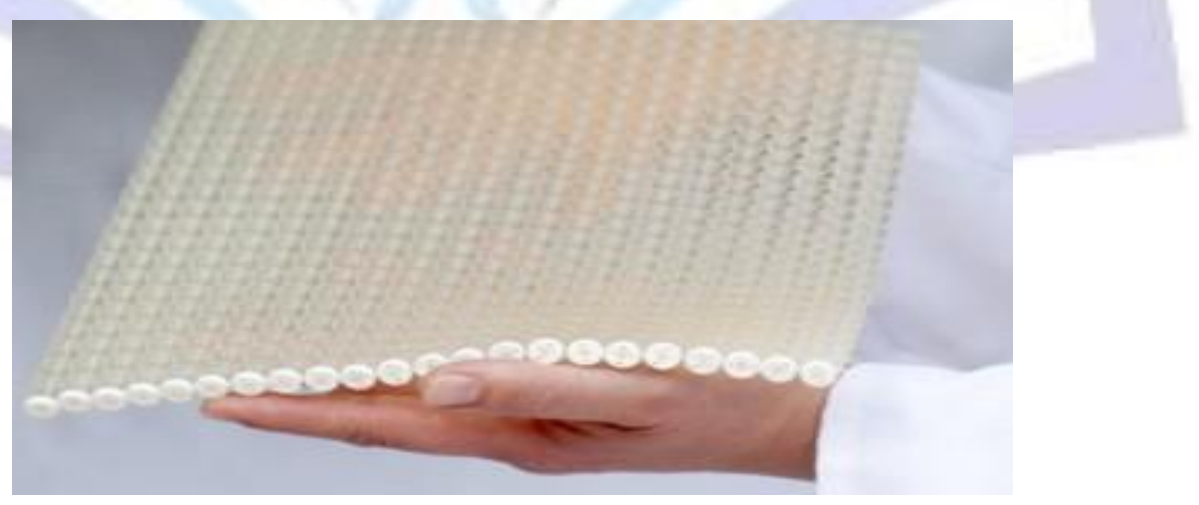

Fig 1: The Freiburg Flap Applicator Set

The Freiburg Flap Applicator is used in conjunction with the OncoSmart flexible implant tubes which are used to guide the source to the points of planned implant.Fixed slabs of RW3 (Perspex) - Slabs made of Water-equivalent material (Goettingen White Water) - were added below and above the 2D-array to provide full scattering conditions. The phantom was scanned on computed tomography (CT) for the treatment planning -figure 2- with 2.5-mm slice thickness and the scanned images are exported to the Oncentra Master Brachy treatment planning system for performing different test plans. 


\section{ISSN 2277-3061}

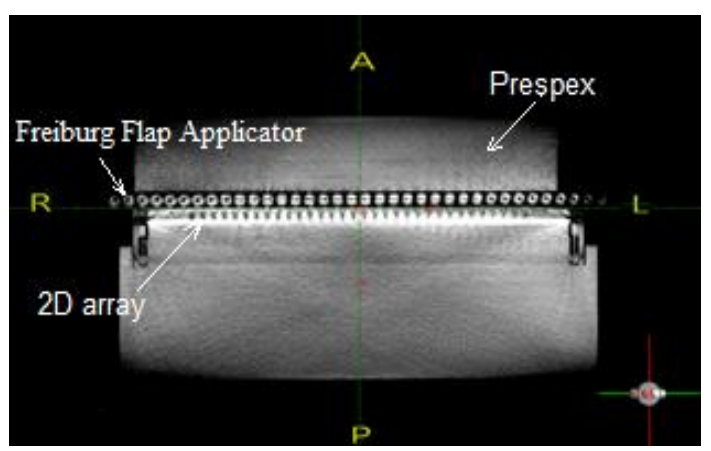

Fig 2: Computed tomography scanned axial image of the measurement phantom

Based on the CT data of the phantom, three different plans imitating three different intracavitary brachytherapy treatment applications were calculated by the planning system. The first plan consists of two active channels to imitate the Fletcher GYN applicator set for vault application -figure 3- and planned to deliver 2 Gy at $1 \mathrm{~cm}$ (point A) lateral to the central axis mid the two transfer tubes. The second plan consists of three active channels to imitate the Fletcher GYN applicator set for full application -figure 4- and planned to deliver 2 Gy at $2 \mathrm{~cm}$ (point A) lateral to the tandem-like transfer tube central axis and at $4 \mathrm{~cm}$ lateral to that axis at the top level of the two other transfer tubes. The third plan consists of only one active channel to imitate the Fletcher GYN applicator set for cylinder application -figure 5- and planned to deliver $2 \mathrm{~Gy}$ at $2 \mathrm{~cm}$ (point A) lateral to the central axis of the cylinder-like transfer tube.

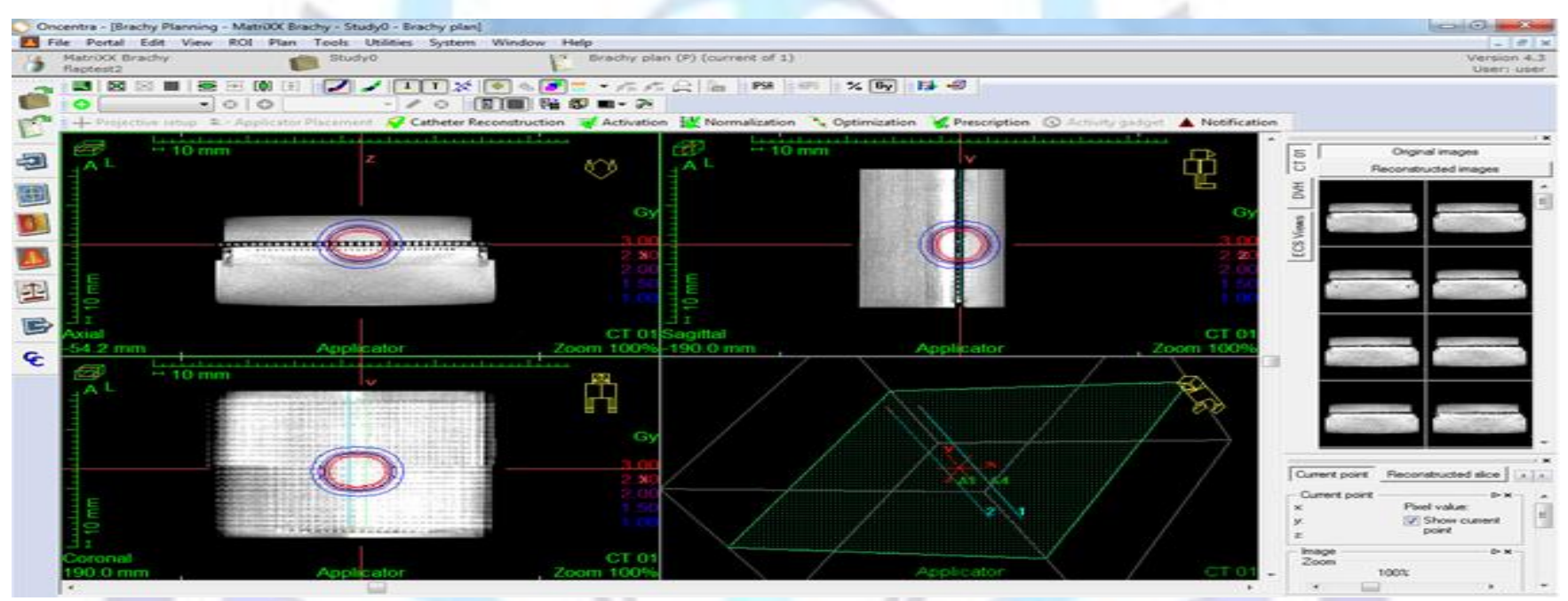

Fig 3: Oncentra master plan brachy short-cut of the vault test case

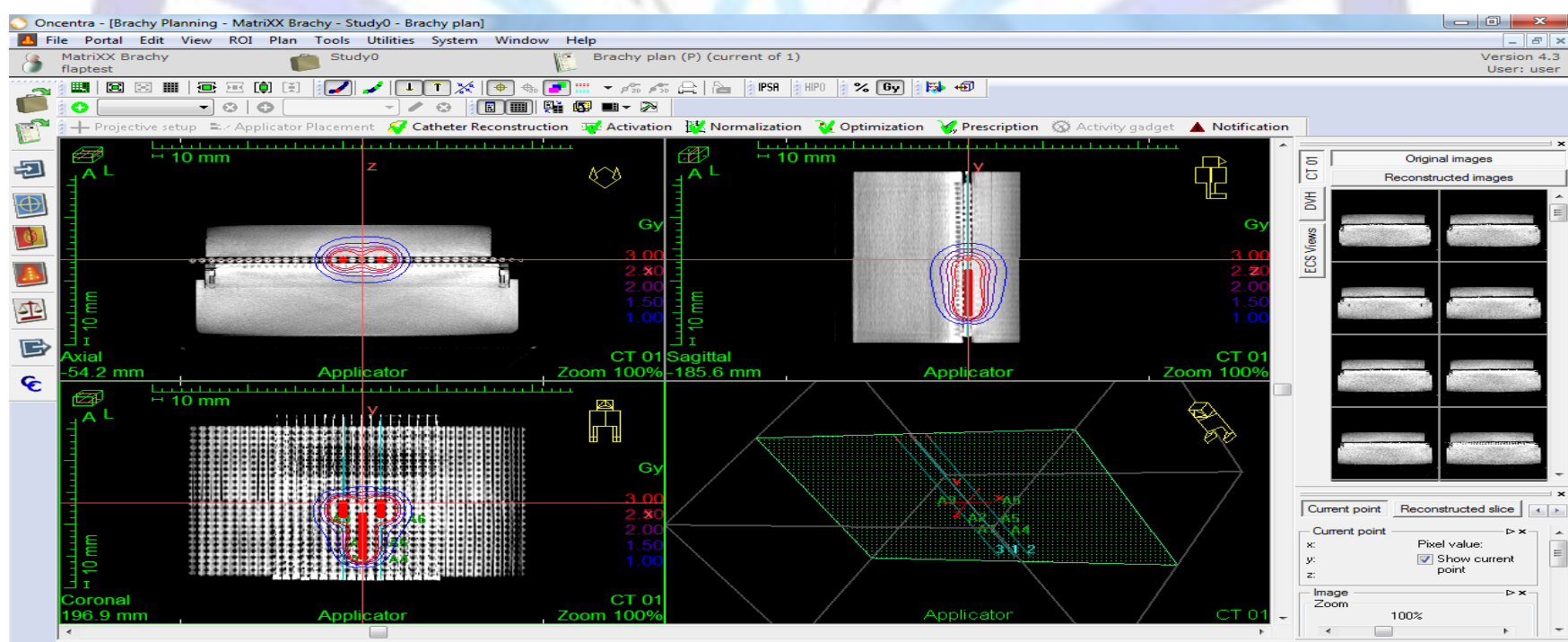

Fig 4: Oncentra master plan brachy short-cut of the full test case 


\section{ISSN 2277-3061}

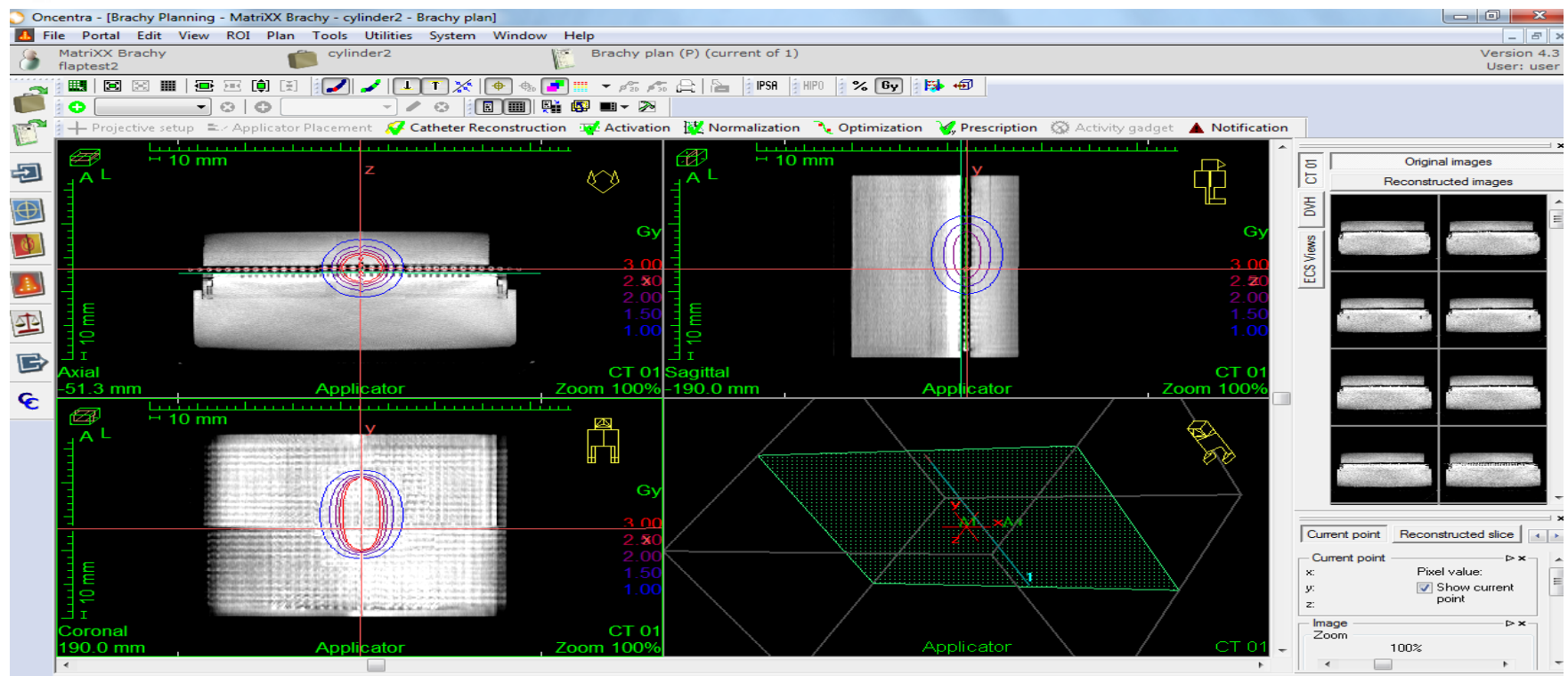

Fig 5: Oncentra master plan brachy short-cut of the cylinder test case

The treatment plans were then exported to theconsole of the MicroSelectron HDR afterloader (version 3, Nucletron BV) which contains the Ir-192 sourcefor practical application on the measurement phantom. The ion chamber array was irradiated, setting the position of the detector plane of measurement to correspond with the computed plan. The TPS generated plans were also exported to the VeriSoft software for comparison with measured data. For comparison of dose distributions, both dose planes were normalized to the global maximum dose of the reference matrix (measured data set). The calculated and measured dose distributions were then compared using the Gamma index method [9] where both plans were normalized to the maximum dose ofthe reference matrix (measured data set), this normalization decreases the possibility of miss matching between measured and calculated values hence gamma passing rate results increase. Gamma indexes were evaluated using a dose-difference criterion of $3 \%$ and a distance criterion of $3 \mathrm{~mm}(\gamma \leq 1)$.

\section{RESULTS}

Figures 6-11 show comparisons of measured and TPS-calculated dose distributions for the three test patterns. Comparisons of dose distributions were done using Gamma 2D global method [9] where the passing criterion is $y \leq 1$ (with $3 \%$ delta dose and 3-mm distance criteria).Tables 1-3 illustrate the comparison points' statistics for the three test applications. Such that the total number of evaluated dose points for the vault test case (table 1) is 9755 , $98.6 \%$ of them (9623 point) passed the criteria of acceptability ( $3 \%$ delta dose and $3-\mathrm{mm}$ distance criteria) and $1.4 \%$ of them (132 point) failed it.The total number of evaluated dose points for the full test case (table 2) is 19964,93.6\% of them (18683 point) passed the criteria of acceptability and $6.4 \%$ of them (1281point) failed it.And the total number of evaluated dose points for the cylinder test case (table 3 ) is $19871,96.9 \%$ of them (19258 point) passed the criteria of acceptability and $3.1 \%$ of them (613 point) failed it.

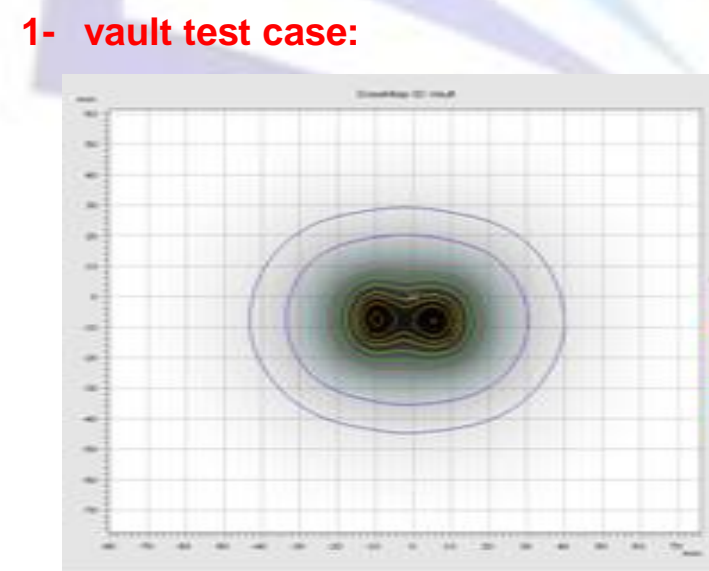

(a)

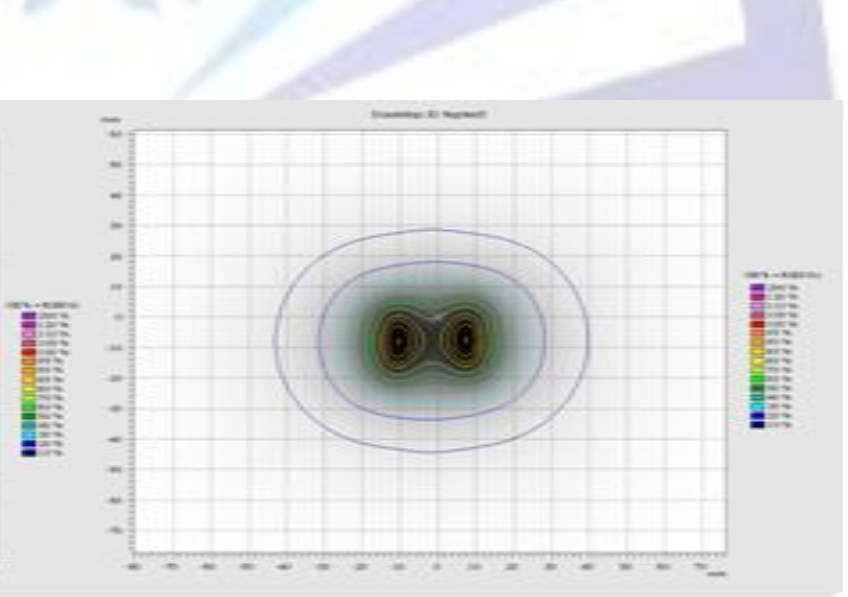

(b)

Fig 6: (a) Dose distribution for measured plan, (b) for calculated plan 


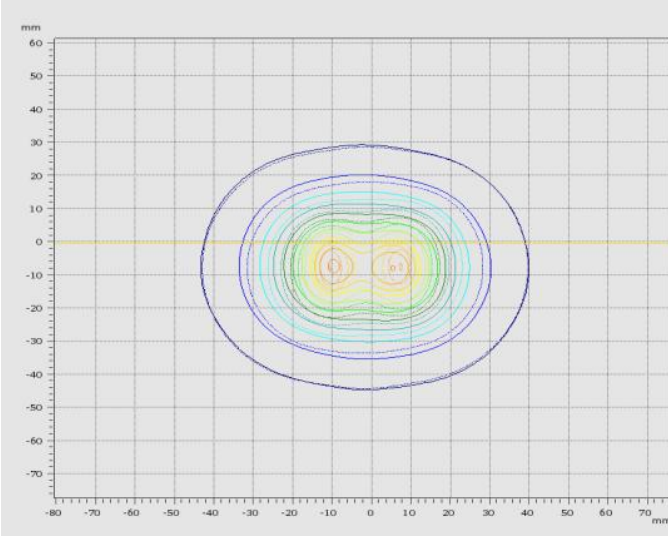

(a)
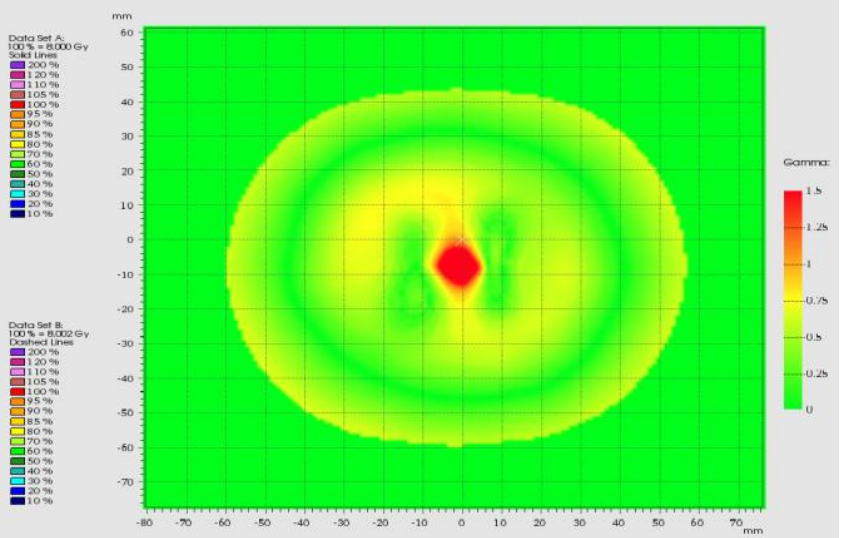

(b)

Fig 7: (a) Isodose comparison between the measured and calculated plans. (b) Gamma distribution of the comparison for the vault test case

2- Full test case:

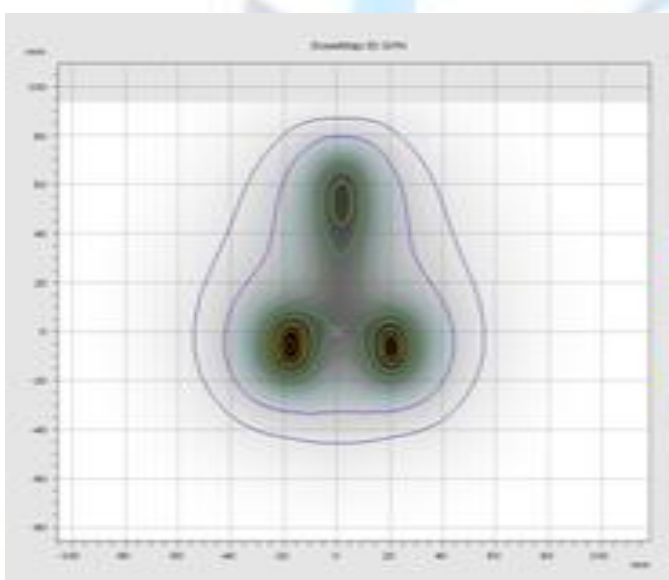

(a)

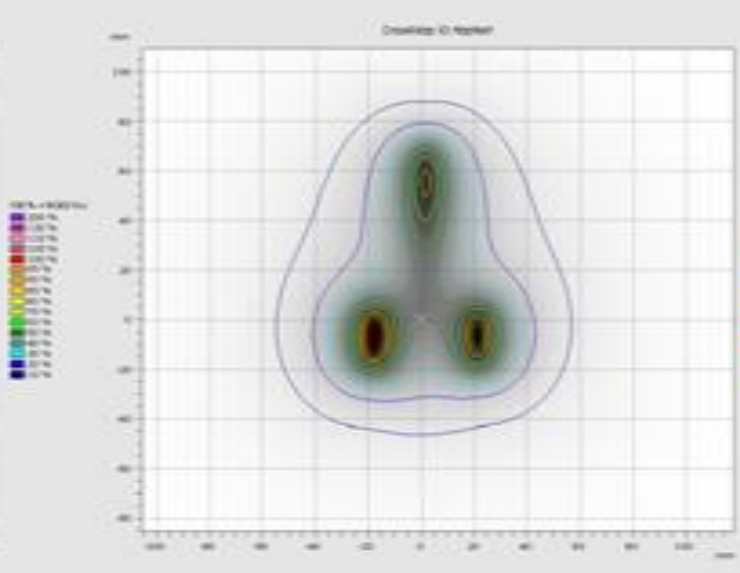

(b)

Fig 8: (a) Dose distribution for measured plan, (b) for calculated plan

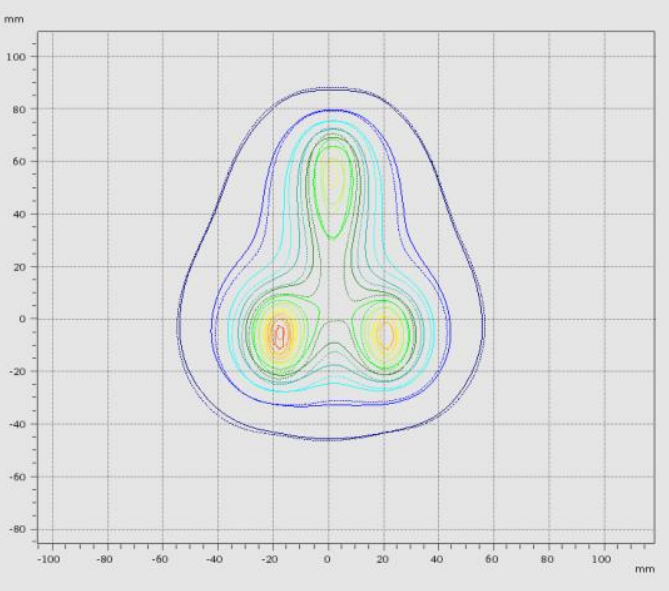

(a)

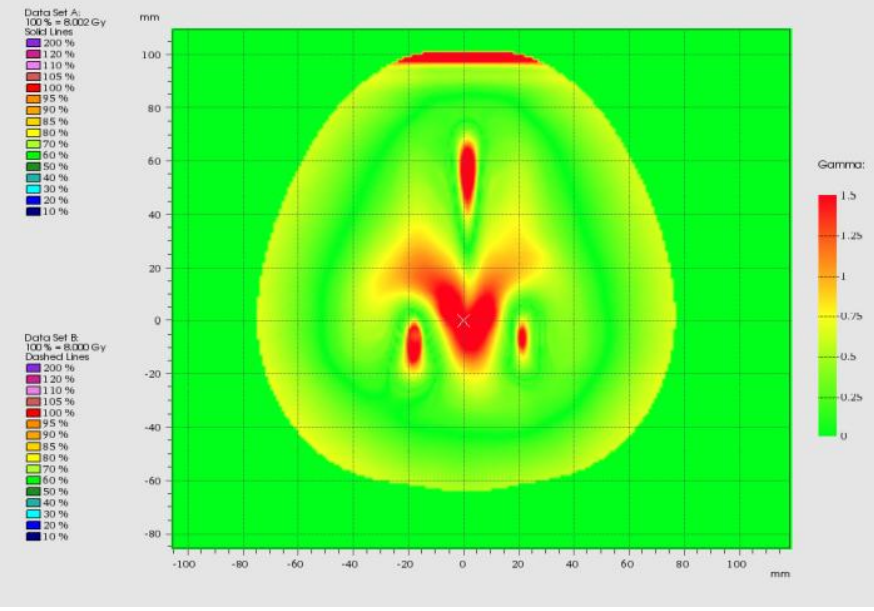

(b)

Fig 9: (a) Isodose comparison between the measured and calculated plans. (b) Gamma distribution of the comparison for the full test case 


\section{ISSN 2277-3061}

3- cylinder test case:

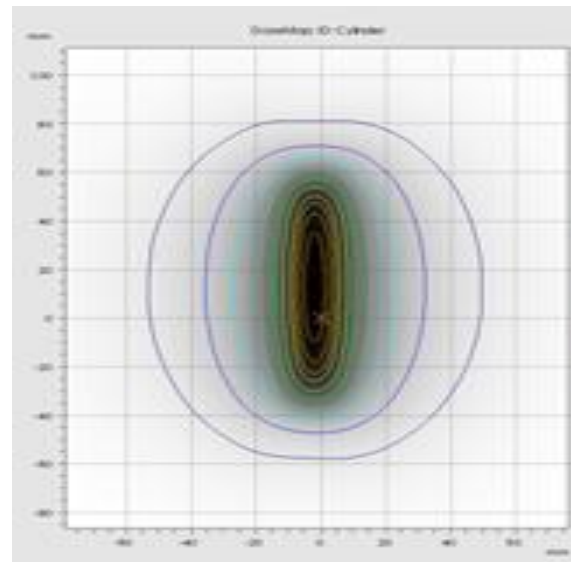

(a)
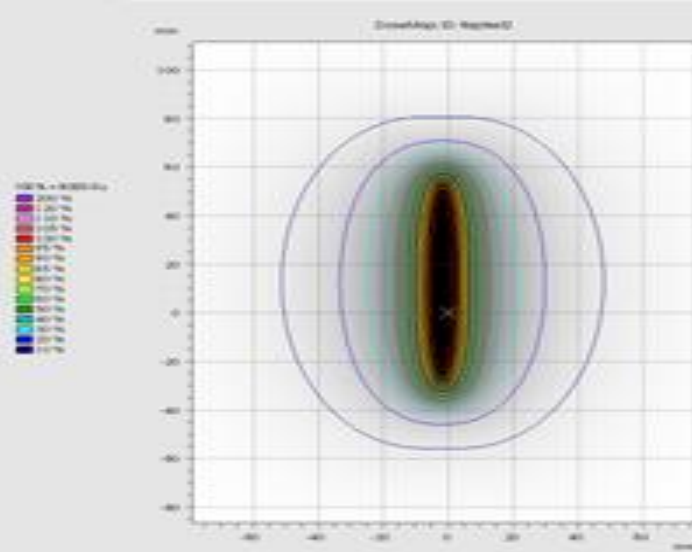

(b)

Fig 10: (a) Dose distribution for measured plan, (b) for calculated plan

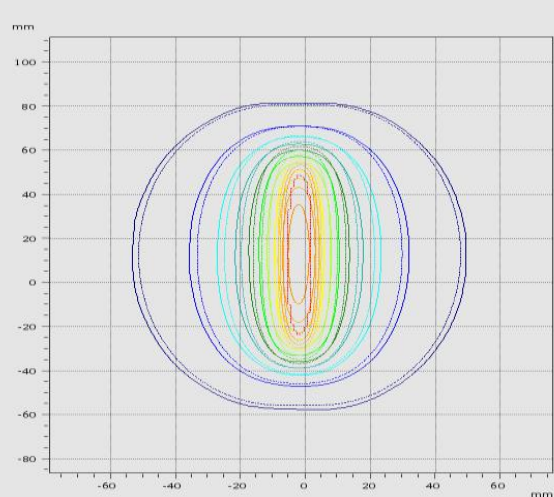

(a)
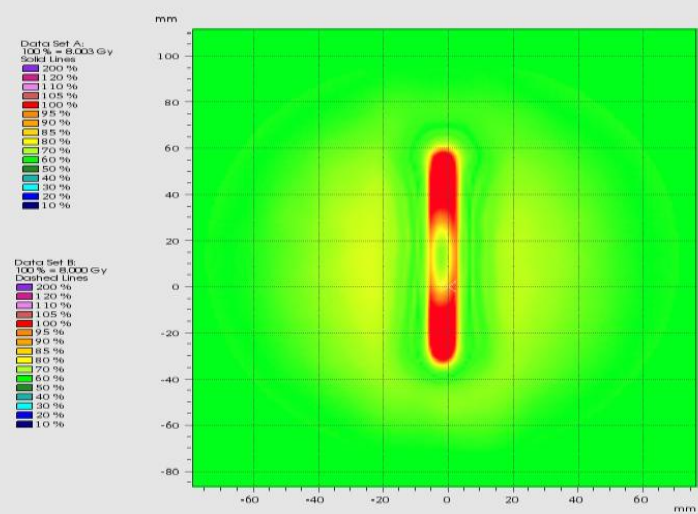

(b)

Fig 11: (a) Isodose comparison between the measured and calculated plans. (b) Gamma distribution of the comparison for the cylinder test case

Table 1. The comparison points' statistics for the vault test application

\begin{tabular}{|c|c|c|}
\hline \multicolumn{3}{|c|}{ Statistics } \\
\hline & Number & $\%$ \\
\hline Evaluated dose points & 9755 & 100 \\
\hline Passed & 9623 & 98.6 \\
\hline Failed & 132 & 1.4 \\
\hline
\end{tabular}

Table 2. The comparison points' statistics for the full test application

\begin{tabular}{|c|c|c|}
\hline \multicolumn{3}{|c|}{ Statistics } \\
\hline & Number & $\%$ \\
\hline Evaluated dose points & 19964 & 100 \\
\hline Passed & 18683 & 93.6 \\
\hline Failed & 1281 & 6.4 \\
\hline
\end{tabular}


Table 3. the comparison points' statistics for the cylinder test application

\begin{tabular}{|c|c|c|}
\hline \multicolumn{2}{|c|}{ Statistics } \\
\hline & Number & $\%$ \\
\hline Evaluated dose points & 19871 & 100 \\
\hline Passed & 19258 & 96.9 \\
\hline Failed & 613 & 3.1 \\
\hline
\end{tabular}

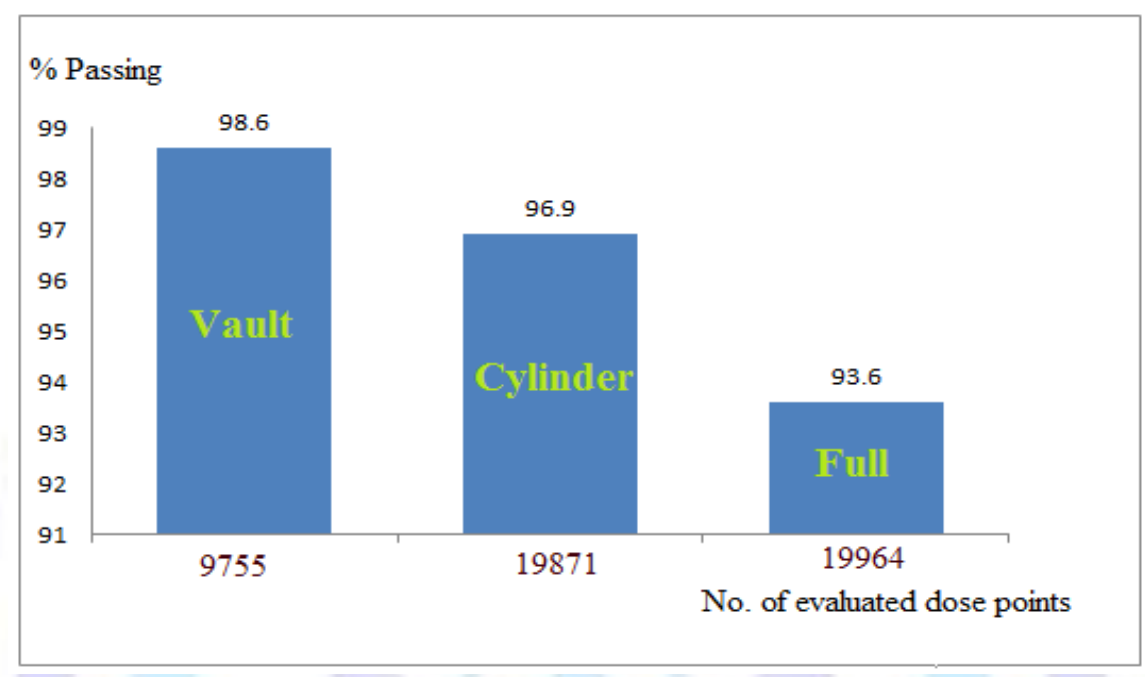

Fig 12: A chart presentation relating the number of evaluated dose points with the passed percentage

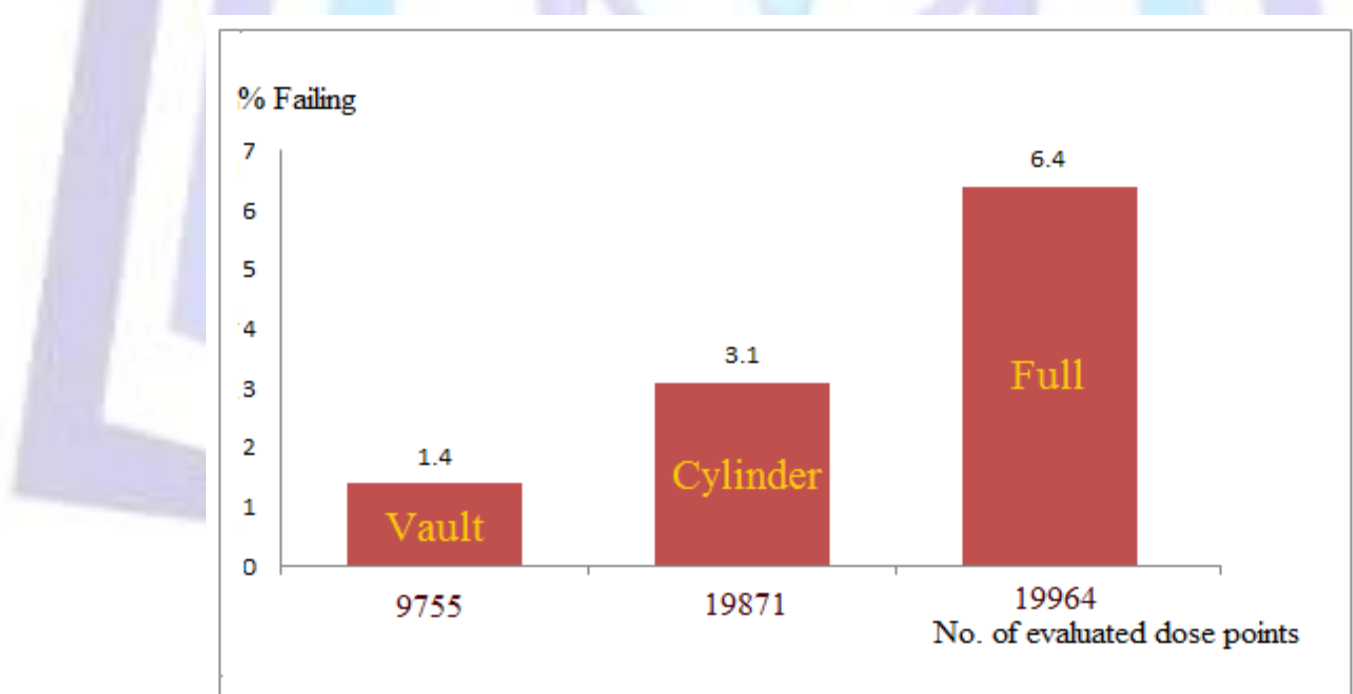

Fig 13: A chart presentation relating the number of evaluated dose points with the failed percentage

\section{DISCUSSION}

Comparisons were made with $3 \%$ delta dose and $3-\mathrm{mm}$ distance criteriawithin the VeriSoftsoftware\&both dose planes (measured and calculated) were normalized to the global maximum dose of the reference matrix (measured data set). The percentage of the number of points passedthe dose distribution comparisonin the vault test case was98.6\%, in the full test case was $93.6 \%$ and in the cylinder test case was $96.9 \%$. Relating these passing percentage values to the number of the evaluated dose points for each test case (figures $12 \& 13$ ); it can be found that as the number of these pixels increases the possibility of having more failing points increases.

More likely to be illustrated in the full test case whose the maximum number of dose points (19964) and the highest failing percentage $(6.4 \%)$ of its total evaluated dose points, and in the vault test case whose the minimum number of dose points $(9755)$ and the lowest failing percentage $(1.4 \%)$ of its total evaluated dose points. The relation between the passing 
percentage of the comparison and the number of the total evaluated dose points can be interpreted to the comparison matrix area which when is increased (increased number of pixels evaluated) the possibility to have more failing points is increased and vies versa for smaller areas.

\section{CONCLUSION}

The array measurement technique has been successfully validated for three different test cases (vault, full and cylinderlike applications) against TPS dose distribution calculations. The 2D chamber array can be applied as a routine quality control toolproviding reliableverification toolof the actual dose distribution delivered by HDRtreatment equipment for the different test cases. The comparisons made led to make a relation between the passing percentage values to the number of the evaluated dose points for each test case and it was found that as the number of these pixels increases the possibility of having more failing points increases.

\section{REFERENCES}

[1] Nath, R.; Anderson, L.L.; Luxton, G.; et al. Dosimetry of interstitial brachytherapysources: Recommendations of the AAPM Radiation Therapy Committee TaskGroup. Med. Phys. 22:209-34; 1995.

[2] Jeffrey F. Williamson. The sievert integral revisited: Evaluation and extension to125I,169Yb, and 192/r brachytherapy sources. International Journal of Radiation Oncology Biology Physics; Volume 36, Issue 5, 1 December 1996, Pages 1239-1250.

[3] Wang R, Sloboda RS. Monte Carlo dosimetry of the VariSource high dose rate 192 Ir source.Med Phys.1998;25:415423.

[4] Devic, S.; Tomic, N.; DeBlois, F. Commissioning of brachytherapy TPS using EBT Model GafChromic Film. Med. Phys. 36:4323; 2009.

[5] Niroomand-Rad, A.; Blackwell, C.R.; Coursey, B.M.; et al. Radio-chromic film dosimetry: Recommendations of AAPM Radiation Therapy Committee Task Group 55.Med. Phys. 25;2093-115; 1998.

[6] Herzen, J.; Todorovic, M.; Cremers, F.; et al. Dosimetric evaluation of a 2D pixelionization chamber for implementation in clinical routine. Phys. Med. Biol. 52:1197-208; 2007.

[7] Saminathan, S.; Manickan, R.; Chandraraj, V.; et al. Dosimetric study of 2D ionchamber array matrix for the modern radiotherapy treatment verification. J. Appl.Clin. Med. Phys. 11:116-27; 2010.

[8] Mammo Yewondwossen. Characterization and use of a 2D-array of ion chambers for brachytherapy dosimetric quality assurance.Medical Dosimetry 37 (2012) 250-256.

[9] Low, D.A.; Harms, W.B.; Mutic, S.; et al. A technique for the quantitative evaluation of dose distributions. Med. Phys. 25:656-61; 1998.

\section{Author' biography with Photo}

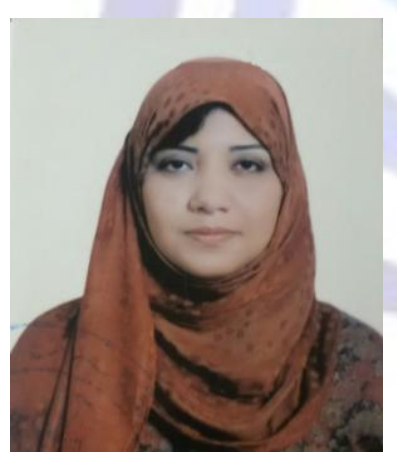

Rasha Moustafa was born in Cairo, Egypt in 1985. She received her B.Sc. and M.Sc. degree both in biomedical physics from Helwan University in 2006 and 2013 respectively. She is doing her Ph.D. degree in biomedical physics from Helwan University faculty of Science during this year (2016). Currently, she is a medical physicist at the radiotherapy department ,national cancer institute of cairo, Egypt. Her research interests radiotherapy planning, Brachytherapy, radiation therapy, Dosimetry. 